\title{
GENERAL APPROACHES TO IMPROVING ANTICORROSION AND ANTIFOURING COATINGS
}

\author{
${ }^{1}$ Veniamin BOLDYREV, ${ }^{2,3}$ Vladimir MEN'SHIKOV, ${ }^{2}$ Anastasiya SAVINA, 1,2Nikita BOGATOV, \\ ${ }^{2}$ Aleksandr ZOTKIN, ${ }^{3}$ Flura IL'DARHANOVA, ${ }^{1}$ Anastasia RAZVODOVA \\ ${ }^{1}$ Bauman Moscow State Technical University, Moscow, Russia \\ ${ }^{2}$ D. Mendeleev University of Chemical Technology of Russia, Moscow, Russia, \\ savina bmstu@inbox.ru
}

${ }^{3}$ Scientific Production Association "Lakokraspokrytiye», Khotkovo, Moscow region, Russia

https://doi.org/10.37904/metal.2020.3571

\begin{abstract}
Metal structures, equipment, ships, port and hydraulic structures, other objects operating in river or sea water, after some time are exposed to the inhabitants of the aquatic environment. The surface is overgrown with algae; various small marine organisms attach to it. Fouling with algae and microorganisms becomes the reason for the intensification of metal corrosion processes in an aggressive aqueous medium, which leads to premature destruction of objects.
\end{abstract}

Bio-fouling creates a number of problems for operation - from reducing the fuel efficiency to losing the design. Only recorded losses from biodeterioration account for $5-7 \%$ of the value of world industrial products, and they are gradually growing.

Keywords: Corrosion, antifouring, biocides, paints and varnishes

\section{FOULING PROBLEM}

The economic and environmental consequences of biocorrosion can be quite noticeable if we take into account the following negative facts that occur during fouling:

1) high cost of docking the ship (over 1 million euros per day).

2) the ship serves with less efficiency and safety.

3) when the ship is fouling, fuel consumption increases by more than $40 \%$.

4) aggressive species of fouling can invade new ecosystems and cause damage to these systems.

In connection with the foregoing, the protection of structures from biological damage and fouling is of great importance.

It is common practice to prevent fouling by algae and marine organisms - painting the surface of such objects with special paints and varnishes (paintwork materials), which are divided into the following categories: biocidal contact action and preventing fouling (self-cleaning) [1-2].

Biocidal coatings include antifoulants - agents that inhibit biological fouling due to their high toxicity. Anti-fouling paints and varnishes that do not contain antifoulants include the use of a polymer soluble in seawater with a strictly controlled polishing rate and an increase in the mechanical strength of these materials.

Previously, the problem of anti-fouling was successfully solved by the use of self-cleaning coatings with controlled release of tributyltin toxin. Biocide was extremely effective. This toxic and persistent material has been used so widely that its accumulation in the marine ecosystem has become environmentally unsafe. The International Maritime Organization for the Control of Harmful Antifouling Systems on Ships (IMO) has adopted 
the International Convention on the Limitation, since 2003, of the use of antifouling systems containing tributyltin and other organotin compounds. Since 2008, these biocides have been banned.

In connection with this circumstance, new types of paints and varnishes began to be developed, primarily selfpolishing anti-fouling coatings that did not contain organotin biocides, with a strictly controlled polishing rate. Rosin was used as a self-polishing film-forming base, and copper oxide and other copper-containing compounds are used as the main biocide in such compositions. Organic biocides such as diuron and cineb are also used in combination with copper oxide. However, the service life of such coatings between docks, even in the case of reinforcing fibers, is a maximum of 5 years, and in normal practice - 2-3 years, which is associated with the mechanism of action of the coating: dissolving the polymer in water to obtain a polishing effect.

In addition, copper is less toxic than tributyltin, ecologists predict that for it, ultimately, it will be necessary to look for an alternative in 2-3 years.

Thus, if there is a current ban on the use of organotin antifouling substances and restrictions on the use of other biocidal materials, there is no certainty regarding the acceptable dates for the use of copper in antifouling coatings. Therefore, the development of low- or non-toxic anti-fouling coatings is relevant.

Modern environmental requirements in the development of new anti-corrosion and anti-fouling coatings are as follows:

1) the complete prohibition, in accordance with the decision of the UN (IMO Convention), of the use of tincontaining antifouling coatings, as the most dangerous for the environment.

2) development and use in anti-fouling coatings of new low-toxic biocides that do not contain heavy metals.

3) creation of antifouling coatings, the toxic components of which in seawater quickly (for example, within no more than 12 hours) lose their biological activity.

4) development of coatings with delayed release of harmful components from them into the environment, which will ensure a decrease in concentration in sea water.

There are two main approaches to meeting the above requirements:

1) creating a very slippery coating surface to which marine organisms cannot attach.

2) use of biodegradable biocides in coatings, which will slowly leach out of the coating.

\section{METHODS OF PROTECTION AGAINST CORROSION AND BIOFOULING OF SURFACES}

Thus, based on the above requirements, there are two directions in the development of protection against corrosion and biofouling of objects operating under the influence of fresh and sea water:

1) the creation of coatings with reduced surface energy - hydrophobic, with a slippery surface that prevents fouling (adhesion to micro-and macroorganisms on the paintwork), so biocidal additives are not added to such coatings (non-biocidal protection).

2) the use of new highly effective environmentally friendly biocides with reduced toxicity and an adjustable rate of leaching of the biocide, which can significantly increase the life of the biocide coating.

Non-biocidal protection is a new approach to maintaining the cleanliness of the underwater surfaces of ships, it consists in the use of non-toxic coatings, from which the layering is easily removed mechanically, often simply by oncoming water when the ship is moving. This biofouling coating technology is based mainly on the surface chemistry of siloxanes, to which bioorganisms loosely adhere. The composition of such coatings usually includes polydimethylsiloxane with functional silanol groups $(\mathrm{SiOH})$, silicon dioxide, catalysts and silanes and alkoxyl groups or a silicate crosslinking agent. Due to their smoothness and low surface energy, marine organisms do not adhere to such a coating [3]. The increased smoothness of the coating also improves the speed characteristics of ships and provides fuel savings [4-5]. 
The main film-forming agents of such paint and varnish compositions are silicone elastomers, including fluorinated, as well as medium molecular weight fluoroepoxy resins, cured by amino-silicone compounds and forming coatings with excellent physical, mechanical and protective properties, ensuring a long service life when used in water and protecting surfaces from biofouling [6]. Fluorinated or silicon additives are widely used as a means of reducing surface or interfacial tension in coatings at low amounts of administration, since they easily migrate to the surface during curing.

But coatings of this type are effective for use as anti-fouling substances on fast moving ships, where streams of water allow washing away attached organisms. The general rule is that in order to make these coatings effective, you need a speed exceeding 18 knots, which is why they are used on high-speed vessels.

Exceptionally low surface tension coatings or coatings are known which slowly leach siloxane components, so that the surface remains active.

The difficulties encountered in the production of coatings of this type include the ability of the coating surface to change over time. This is expressed in the fact that the surface tension on a surface freshly moistened with clean water can differ significantly from the surface tension on a surface that has been immersed in water for several days.

Such a change in surface properties results in a decrease in interfacial tension, which in turn allows microorganisms to more easily attach to the coating. The scale and rate of such a change depends on the composition of the coating: they are lower than hard, highly cross-linked coatings.

Their industrially produced non-biocidal anti-fouling coatings using silicone technology are known for Intersleek coatings from AkzoNobel and Hempasil X3 from Hempel, SeaLion from Jotun, which are characterized by low surface energy and a smooth coating surface.

An increase in biostability is possible through the use of new highly effective environmentally friendly biocides with reduced toxicity.

Coatings based on currently used enamels in the initial period of operation are characterized by an excessively high rate of leaching of toxins, with about $25-30 \%$ of toxins being consumed unproductive. When developing coatings with a controlled biocide leaching rate, one of the most promising ways to create anti-fouling coatings is the use of diffusion surface layers. In this case, the hydrophilicity of the surface layer and its porosity can be controlled by changing the ratio of hydrophilic and hydrophobic components in the film. Hydrophilic components can have different solubilities, vary widely over the size of the molecular weight, in some cases they can serve as toxins.

Both in non-biocidal coatings and in coatings with biocides, the use of nanotechnologies is promising, for example, the production of nanostructured surfaces, or the use of nanosubstances acting at the nanoscale. A successful solution to the problem of obtaining low-toxic biocides is possible in the transition to nanotechnology, which will initially allow controlling the particle morphology at the micro level. A nanosubstance is characterized by a special distribution of atoms and electrons in it, which gives the nanoparticle substances special properties (minimum volume, maximum surface energy, special energy, etc.) in contrast to compounds of a similar chemical structure with large particles. Abroad known anti-fouling paint systems that do not contain biocides, with a nanostructured surface, which effectively resists fouling by marine organisms. Antifouling paint systems for marine conditions were developed on the basis of fluoropolymer, epoxy resin, nanosized titanium dioxide, silicone and acrylic polymers with radicals containing $\mathrm{Cu}$ and $\mathrm{Zn}$. There is evidence of compositions containing nanosized silica to prevent biofouling [7-10].

It is also possible to use epoxy siloxane hybrid binders filled with nanocomponents in the anti-fouling materials. For example, coatings $375 \mathrm{~nm}$ thick formed by $\mathrm{TiO}_{2}$ nanoparticles (anatase) with diameters of 15-18 nm were prepared on the stainless steel surface by the sol-gel method and were hydrophobized with a self-ordered layer of fluoroalkylsilane (the contact angle of water-wetting of the hydrophobized coating was $150^{\circ}$ ). The 
surface morphology and coating structure were studied by scanning electron microscopy and X-ray phase analysis. According to electrochemical measurements, the coatings showed excellent corrosion resistance in a chloride solution at room temperature [11].

\section{HYPOTHESES AND SUGGESTIONS}

One of the new directions in the use of low toxic biocides, according to foreign publications, is the use of a number of standard pharmaceutical products for use as antifouling agents. Note that it is not necessary to kill marine organisms, it is enough not to let them sit on the surface of the coating. It turned out that the group of products that were investigated are called ketimines and which are known to have low toxicity due to their pharmaceutical use, can be used as biocides. Medetomidine is especially effective; A system for the prolonged delivery of a drug substance was developed, the action of which is based on the adsorption of medetomidine on the surface of metal oxide nanoparticles. However, these biocides are in the initial stages of development and are only examples on the way to creating coatings that are not harmful to the environment and do not contain toxic substances.

\section{REFERENCES}

[1] KARPOV V.A. and others. An integrated approach to protection against marine fouling and erosion. KMK Scientific Press Ltd., 2007.

[2] LOMAKINA G.YU. Role of biofilms in microbiologically influenced corrosion of metals. Herald of the Bauman Moscow State Technical University, Series Natural Sciences, 2020, no. 1, pp. 100-125.

[3] VITUKI Gerall L. Evolution of silicone technology in coatings. Eurasian Chemical Market, 2017, no. 1, pp. 96-102.

[4] BOGOMOLOV, B.B., ZUBAREV, A.M., MESHALKIN, V.P., MENSHIKOV, V.V., BOLDYREV, V.S. Intelligent logical information algorithm for choosing energy- and resource-efficient chemical technologies. Theoretical Foundation of Chemical Engineering. 2019, vol. 53, no. 5, pp. 709-718.

[5] FADEEV G.N., BOLDYREV V.S., AVERINA YU.M., BOGATOV N.A. Metal surface treatment in a low-frequency exposure field. Tsvetnye Metally, 2019, no. 10, pp. 73-77.

[6] STOYE D., FREYTAG V. Paints, coatings and solvents. Professija, 2007

[7] CHUGOKU Marine Paints, OYA Masaki, NIKAMURA Naoya, TSUBOI Makota. Antifouling coating composition, coating film therefrom, base material covered with the coating film and antifouling method. US Patent 6916860.

[8] YAMAMORI Naoki, OKAMOTO Satoshi, HIGO Kiyaki, MATSUDA Masayaki. Antifouling coatings. US Patent 7045560.

[9] HUANG Yuan-Chang, SHEEN Yuung-Ching, CHANG Yih-Her, LO Kuo-Feng. Coating material containing hydrophobically-modified particles. British Pat. Appl. 2422608.

[10] ROTH Christoph, SCHONEWERK Jens, BOTTCHER Horst, HINH Le Van. Biofilmhemmende schutzschicht. Patentanmeldung 102004028543.

[11] SHEN G.X., CHEN Y.C., LIN C.J. Study on a hydrophobic nano-TiO2 coating and its properties for corrosion protection of metals. Electrochim. Acta, 2005, vol.50, no 25-26, pp. 5083-5089. 